\title{
2. SIMILITUDE DES CONDITIONS HYDRAULIQUES EN AVAL D'UN SEUIL
}

\author{
2. The similitude \\ of hydraulic conditions downstream of a sill
}

\author{
PAR J. J. VINGÉ, \\ INGÉNIEUR DU LABORATOIRE D'HYDRAULIQUE DE DELFT
}

\begin{abstract}
Pour étudier sur modèle réduit l'érosion et la stabilité de la protection du fond en aval d'un seuil, il faut répondre à deux questions très importantes :

$1^{\circ}$ Est-ce qu'il yl a conformité dans la conflguration et les caractéristiques de la turbulence? $2^{\circ}$ Quand l'érosion se développe, pouvons-nous supposer que la conformité se maintient?

En rapport avec ces problèmes, des recherches systématiques sont en cours; c'est ainsi que l'on fait varier la rugosité et la perméabilité du seuil, de même que les échelles du modele.
\end{abstract}

Ainsi qu'on l'a vu dans une conférence précédente, l'orifice de fermeture dans la partie nord de la digue, dans le Grevelingen, sera fermé suivant une méthode graduelle. Cette méthode est également mise en œuvre pour les très grands orifices de fermeture.

Un point important de l'application de cette

\begin{abstract}
Two very important questions must be answered before a scale model investigation of erosion and bed protection stability downstream of a sill is undertaken. They are the following:-

1. Will the configuration and characteristics of the turbulence on the model be representative of prototype conditions?

2. Will they remain so when erosion develops? Systematic research on these points is now in progress, with sills of varying roughness and permeability, and different model scales.
\end{abstract}

méthode est de savoir si, pendant le rehaussement de la digue, un jet plongeant peut naître.

On entend ici par « jet plongeant» un mouvement d'eau créant un jet concentré qui suit le talus aval de la digue et qui, du côté supérieur, est limité par un tourbillon de surface. Il est évident que ce jet plongeant est très dangereux 

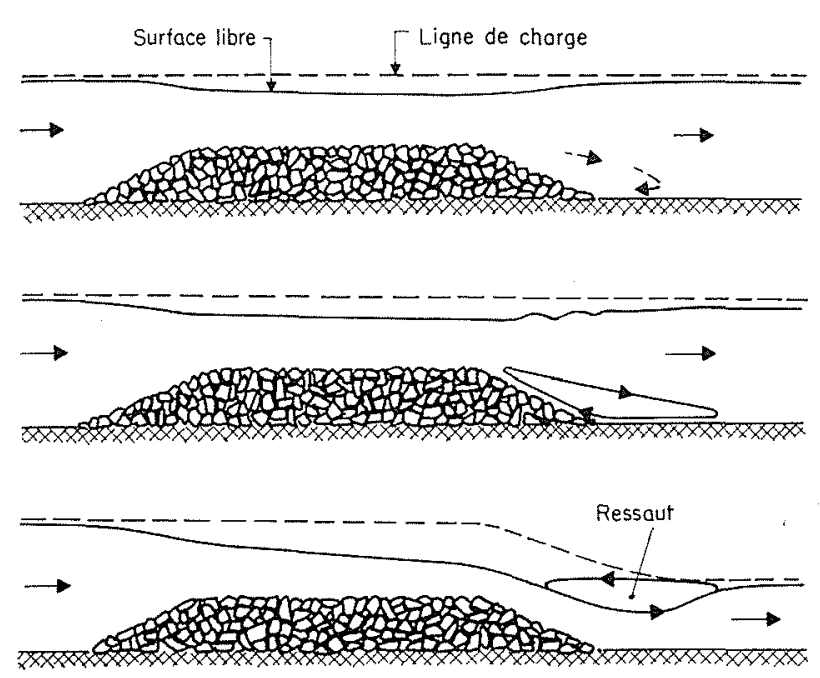

Fig. 1 Types de configurations du courant.

aussi bien pour le talus en aval que pour la parafouille et le fond mobile, et que tout doit être fait pour l'éviter.

Un point important des études sur modèles réduits est de savoir si l'aspect des courants est conforme dans le modèle et dans le prototype.

Les courants qui se produisent lors du rehaussement graduel du seuil dépendent de plusieurs facteurs, qui sont :

- la forme et les dimensions de la digue;

- les hauteurs d'eau en amont et en aval (la chute);

- la rugosité de la digue, et

- la porosité de la digue.

Les courants pouvant se produire sont représentés schématiquement dans la figure 1 pour une digue avec crête horizontale relativement longue. On voit successivement :

1. Courants normaux. Le niveau d'eau audessus de la crête de la digue descend et monte de nouveau un peu en aval par suite de la transformation partielle de l'énergie cinétique en énergie potentielle. Ces courants ne se produisent que lorsque les chutes sont petites. Le tourbillon de fond qui naît n'est que faiblement développé.

2. Sous des hauteurs de chute plus grandes, naît un tourbillon de fond bien développé. Le plan d'eau est alors onduleux en aval. Le ralentissement crée une augmentation de pression, par suite de laquelle le jet le long de la digue se détache et un tourbillon de fond se produit. A mesure que la vitesse augmente, le tourbillon de fond devient plus court.
3. Jet plongeant, avec un tourbillon de surface. L'eau passe par-dessus le talus en aval et il y a de grandes pertes d'énergie. A la ligne de crête en aval, les sous-pressions sont grandes.

Il va de soi que, dans le régime des courants, des formes de transition se présentent.

Un certain nombre de cas dans lesquels on fit varier la rugosité et la porosité de la digue, ont été étudiés, dans un modèle à deux dimensions. Dans chaque cas, le niveau de l'eau en aval a été maintenu constant et la chute (et par conséquent le débit), augmentée graduellement. Ceci a été répété pour un certain nombre de niveaux de l'eau en aval différents.

La figure 2 donne un exemple du résultat qualitatif. Pour quelques niveaux de l'eau en aval, les courants sont schématiquement représentés en fonction de la chute. On distingue des régimes avec des remous an fond, des ressauts (jets plongeants) et des zones transitoires.

Les essais ont montré qu'en utilisant comme matériaux de construction des pierres de 1 à $800 \mathrm{~g}$, la digue s'écroule toujours avant qu'un jet plongeant se produise. Vu la taille des pierres et les vitesses qui se produisent, on peut s'attendre à ce que la viscosité ne joue plus de rôle (grand nombre de Reynolds) et qu'aucun effet d'échelle ne se produise.

Les plus petites chutes sous lesquelles un jet plongeant naît, se présentent quand la digue est lisse et fermée. En faisant la digue plus rugueuse, surtout dans la zone de la ligne de
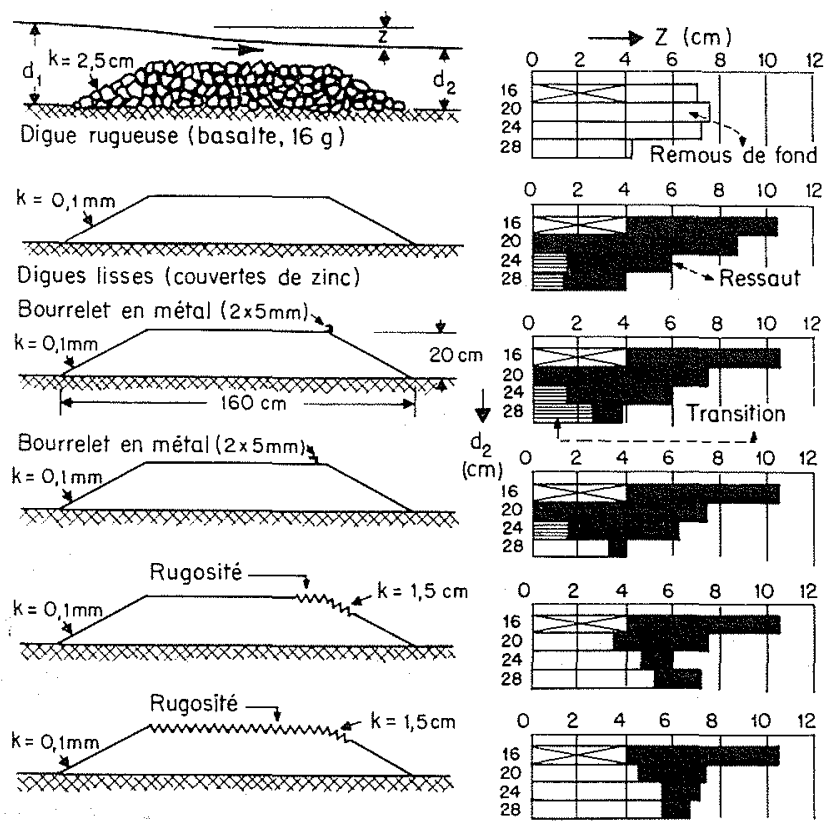

Fici. 2 Situation des recherches qualitatives. 


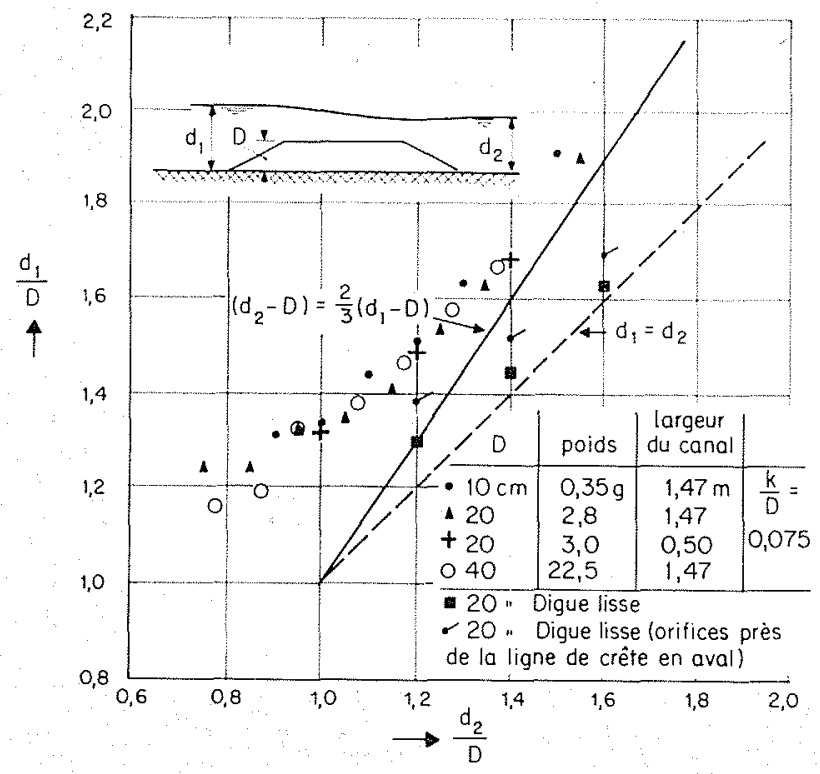

FIG. 3

crête en aval, le jet se décolle plus tôt. Il a paru en outre que l'influence de la rugosité ne peut être représentée en fixant le point de décollement (par exemple à l'aide d'un bourrelet métallique). La répartition des vitesses et celle des pressions sont à peine influencées par la fixation locale.

Si la digue est construite de manière qu'elle soit poreuse, un jet plongeant ne se produit qu'à des chutes plus hautes que lorsque la digue est non poreuse. Ceci provient du fait que, d'une digue poreuse, de l'eau sort aux environs du point de décollement par suite de sous-pressions locales.

En résumé, on peut dire que sous le rapport de la naissance d'un jet plongeant, une digue rugueuse et poreuse est préférable.

A côté de cette interprétation qualitative, il est naturellement d'une grande importance de savoir si les circonstances dans lesquelles un jet plongeant se produit sont, pour une certaine digue, les mêmes dans le modèle et dans le prototype.

Afin de résoudre cette question, une digue courte (largeur de crête $=0$ ) et une digue longue (largeur de crête $=4$ fois la hauteur de la digue) ont été étudiées en faisant varier les échelles de modèle. La première se produit en faisant la fermeture graduelle à l'aide d'un seul téléférique, la deuxième en fermant avec plusieurs téléfériques.

La figure 3 montre les résultats de la digue avec grande largeur de crête. Elle indique la ligne critique suivant laquelle, théoriquement, les courants passent du régime fluvial au régime torrentiel. Cette ligne est une approximation, parce que le facteur $2 / 3$ s'applique à la hauteur d'eau sur la digue. Le niveau d'eau monte encore un peu en aval et par suite, la transition se produira à une hauteur d'eau en aval plus grande.

Les essais font voir que le jet plongeant, dans une digue rugueuse, ne se produit qu'à une hauteur de l'eau en amont plus élevée que celle qui correspond à la hauteur d'eau en amont à laquelle les courants passent du régime fluvial au régime torrentiel. La différence précitée devient plus grande à mesure que la hauteur d'eau en aval est plus faible vis-à-vis de la hauteur de crête. Ceci s'explique par la plus grande influence de la rugosité quand les niveaux de l'eau sont bas.

Cependant, si la digue est achevée lisse, le jet plonge déjà au régime fluvial.

Si la digue est courte, le jet plonge sous des chutes plus petites que si la digue est longue. La cause doit en être cherchée dans la perte supplémentaire par frottement qui se produit pour une digue longue. Pour le reste, les tendances sont les mêmes que pour la digue longue. Une plus grande variation de la hauteur de digue (et proportionnellement du poids moyen des pierres) a été appliquée à la digue courte, mais on constate peu d'effets d'échelle.

De ces séries d'essais avec des pierres ayant un diamètre de $0,7-10 \mathrm{~cm}$, il n'apparaît pas d'influence significative de la viscosité. Le plus petit diamètre correspond pour le Grevelingen à une échelle de longueur de 60.

La figure 4 donne également les conditions

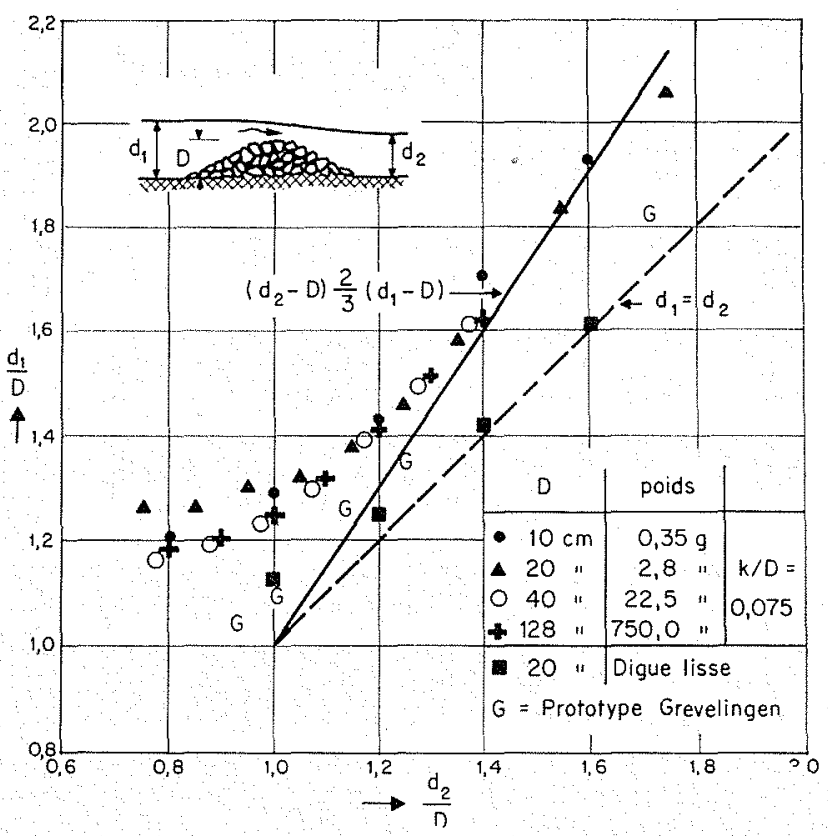

FIG. 4 
d'écoulement dans le Grevelingen. Il paraît, en remarquant qu'il s'agit ici d'une digue rugueuse, qu'il n'y a pas de danger qu'un jet plongeant se produise.

Pour les essais sur modèle à trois dimensions, une représentation exacte des courants est également de grande importance afin d'arriver à une bonne reproduction de l'affouillement.

La configuration horizontale des courants dans des modèles distordus est représentée exactement, à la condition que le facteur d'échelle du frottement $n_{c}$, dans les chenaux aussi bien que sur les barres, soit égal à la racine de la distorsion du modèle.

Cependant, la configuration verticale des courants à un agrandissement de profil, par exemple derrière le seuil d'un orifice de fermeture, n'est pas toujours exactement reproduite dans un modèle distordu. Si, dans la nature, il y a tourbillon de fond, celui-ci est reproduit de facon relativement trop longue dans le modèle. L'influence de ceci sur l'aspect de l'affouillement peut cependant être petite, quand (comme par exemple pour l'orifice de fermeture du Veerse Gat) les affouillements sont causés par des tourbillons à axe vertical.

Des essais détaillés pour des orifices de fermeture dans lesquels la partie à deux dimensions est importante, s'effectuent le plus souvent dans un modèle non distordu à petit facteur d'échelle. L'approche fondamentale du problème de l'affouillement à trois dimensions en est toujours à ses débuts et ne pourra se développer correctement qu'après que, entre autres, le problème

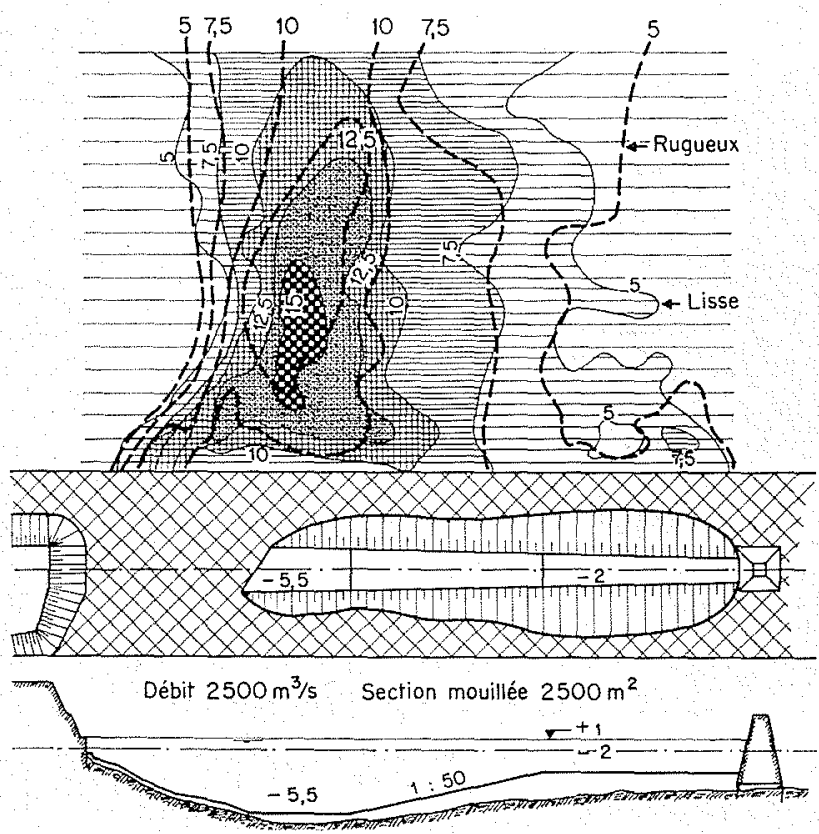

Fig, 5 Erosion Grevelingen après 5 heures modèle.
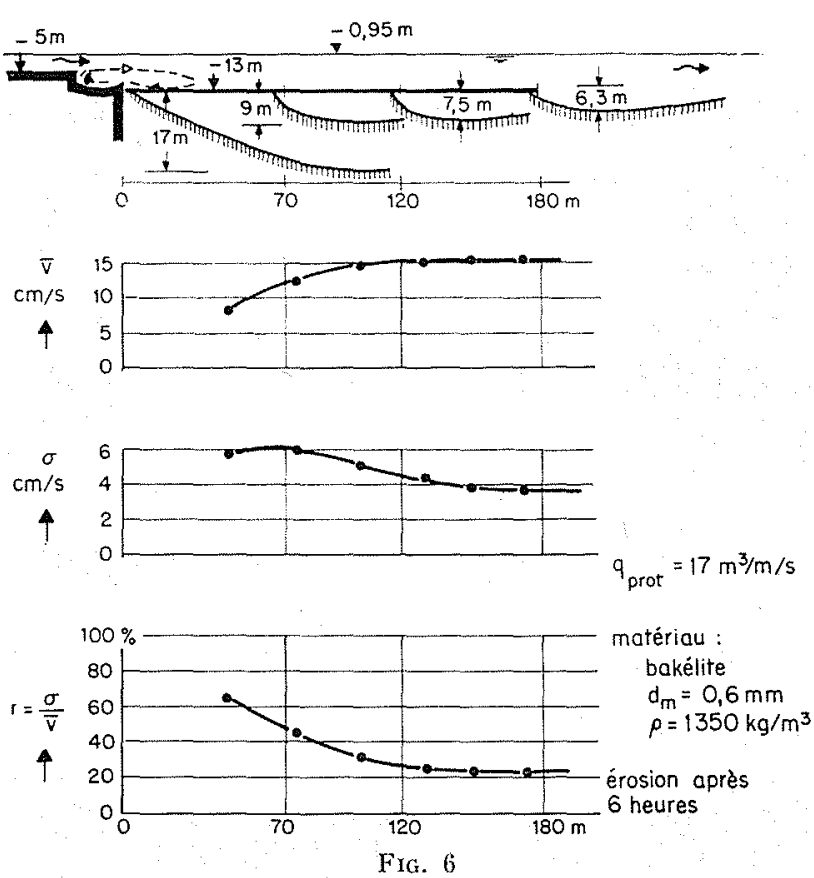

de l'échelle du temps sera résolu à deux dimensions.

Afin d'obtenir un transport suffisant dans les modèles, il est en général nécessaire de réduire le facteur d'échelle pour la vitesse. De ce fait, un agrandissement de la chute se produit par rapport à la nature, et par suite, quand les vitesses sont fortement augmentées, des différences dans le régime des courants et dans la forme de l'affouillement peuvent se produire. Dans ce cas-là, on emploie le plus souvent des matériaux d'un poids spécifique plus léger, grâce auxquels la réduction du facteur d'échelle de la vitesse n'a pas besoin d'être aussi grande.

Le fait que la rugosité de la parafouille est importante lorsqu'il y a érosion apparaît dans la figure 5 qui montre que, pour des circonstances, qui pour le reste sont conformes, la protection du fond a été faite une fois rugueuse et une fois lisse. La protection étant finie lisse, l'érosion est plus grande au milieu du chenal.

Si la protection est achevée rugueuse, la turbulence a déjà diminué davantage quand le courant attaque le fond mobile. La vitesse près du fond, et par conséquent l'attaque, sont alor's plus petites. L'affouillement dépend aussi bien de l'intensité de la turbulence $\left(\sigma=\sqrt{(v-\bar{v})^{2}}\right.$, que de la vitesse moyenne près du fond.

La figure 6 donne un aperçu comparatif des affouillements qui, dans un modèle à deux dimensions, se produisent derrière les écluses d'évacuation dans le Haringvliet. Les longueurs des protections de fond ont été variées. On y voit également les changements de $\sigma, \bar{v}$ et $\sigma / \bar{v}$. 


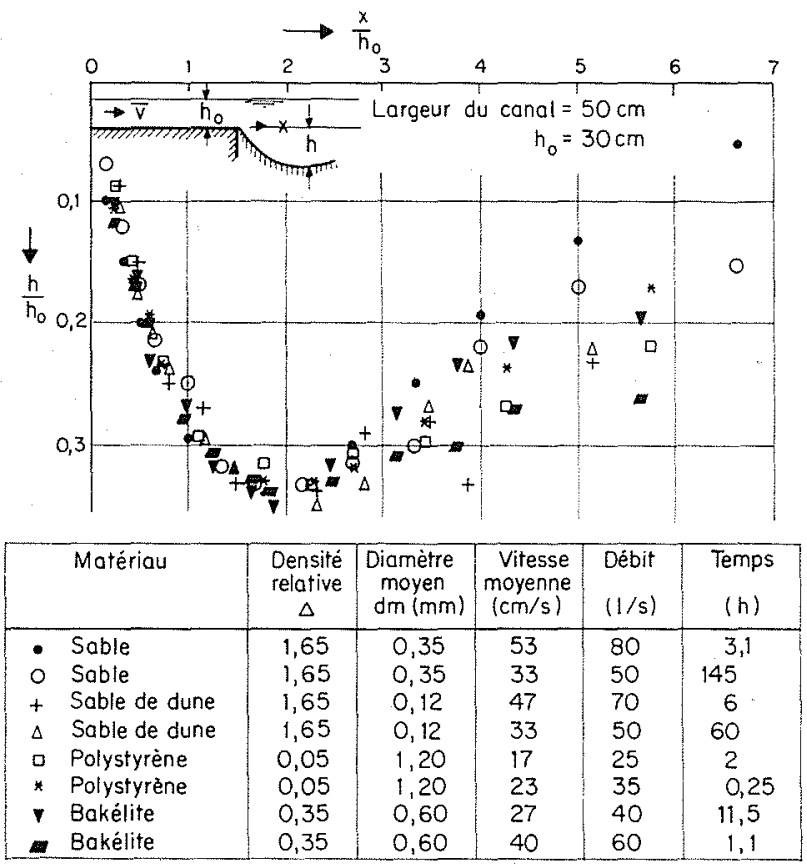

FIG. 7

A une certaine distance derrière le fond de l'écluse, la turbulence $r=(\sigma / \bar{v})$ ne diminue presque plus et c'est le cas également avec l'affouillement.

Partant d'une configuration exacte des courants, les caractéristiques de turbulence pourraient être représentées de façon erronée. Si les courants sont conformes, des différences des caractéristiques de turbulence ne sont possibles que par des effets de viscosité (à exprimer dans le nombre de Reynolds). Aux essais d'affouillement, l'ordre de grandeur du nombre de Reynolds est en général $\mathscr{O}=10^{5}$ et, d'après la littéture, l'influence de la viscosité est alors faible. L'échelle des grands tourbillons est déterminée par la géométrie. Aux plus petits tourbillons, des effets d'échelle se produisent. Dans la technique des mesures on est tenu à des restrictions en mesurant la turbulence, parce que les plus petits tourbillons ne peuvent être mesurés; mais probablement ceux-ci ne sont pas déterminants pour le problème. Plus de recherches pour ce phénomène sont nécessaires.

La question se présente de savoir si la similitude se maintient quand l'érosion se développe.

Partant de la similitude dans la configuration des courants et dans les caractéristiques de la turbulence $(r, \bar{v})$ on peut dire qu'au bout de la parafouille, au temps $t=0$, les conditions de départ exactes existent. Le long du fond de sable, l'intensité du transport est alors conforme à celle dans la nature, pourvu que la puissance à transporter soit supérieure à la valeur critique.

Il découle de l'équation de continuité que $\partial h / \partial t$ est également conforme, c'est-à-dire les changements dans l'affouillement.

La figure 7 donne les résultats d'une série d'essais faits dans un modèle à deux dimensions avec des échelles de vitesse et des matériaux différents. On voit que la forme de l'affouillement est semblable.

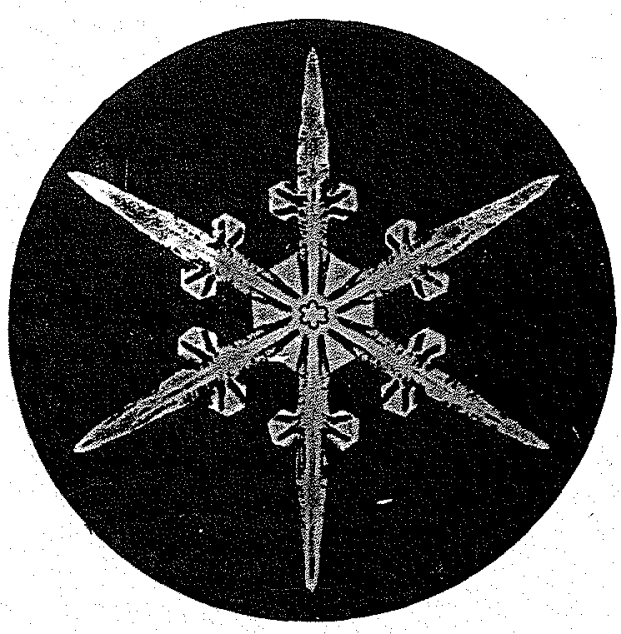

